\title{
Using Deep Learning to Detect Price Change Indications in Financial Markets
}

\author{
Avraam Tsantekidis*, Nikolaos Passalis*, Anastasios Tefas*, \\ Juho Kanniainen $^{\dagger}$, Moncef Gabbouj ${ }^{\ddagger}$ and Alexandros Iosifidis ${ }^{\ddagger \S}$ \\ *Department of Informatics, Aristotle University of Thessaloniki, Thessaloniki, Greece \\ \{avraamt, passalis\}@csd.auth.gr, tefas@aiia.csd.auth.gr \\ ${ }^{\dagger}$ Laboratory of Industrial and Information Management, Tampere University of Technology, Tampere, Finland \\ juho.kanniainen@tut.fi \\ ${ }^{\ddagger}$ Laboratory of Signal Processing, Tampere University of Technology, Tampere, Finland \\ \{moncef.gabbouj, alexandros.iosifidis\}@tut.fi \\ $\S$ Department of Engineering, Electrical and Computer Engineering, Aarhus University, Denmark \\ alexandros.iosifidis@eng.au.dk
}

\begin{abstract}
Forecasting financial time-series has long been among the most challenging problems in financial market analysis. In order to recognize the correct circumstances to enter or exit the markets investors usually employ statistical models (or even simple qualitative methods). However, the inherently noisy and stochastic nature of markets severely limits the forecasting accuracy of the used models. The introduction of electronic trading and the availability of large amounts of data allow for developing novel machine learning techniques that address some of the difficulties faced by the aforementioned methods. In this work we propose a deep learning methodology, based on recurrent neural networks, that can be used for predicting future price movements from large-scale high-frequency timeseries data on Limit Order Books. The proposed method is evaluated using a large-scale dataset of limit order book events.
\end{abstract}

\section{INTRODUCTION}

Using mathematical models to gain an advantage in financial markets is the main consideration of the field of quantitative analysis. The main hypothesis of the field is that the utilization time-series of values like the price and volume of financial products produced by the market can be analyzed with mathematical and statistical models to extract predictions about the current state of the market and future changes in metrics, such as the price volatility and direction of movement. However, these mathematical models rely on handcrafted features and have their parameters tuned manually by observation, which can reduce the accuracy of their predictions. Furthermore, asset price movements in the financial markets very frequently exhibit irrational behaviour since they are largely influenced by human activity that mathematical models fail to capture.
Recently there have been multiple solution to the aforementioned limitations of handcrafted systems using machine learning models. Given some input features machine learning models can be used to predict the behaviour of various aspects of financial markets [1], [2], [3], [4]. This has led several organizations, such as hedge funds and investment firms, to create machine learning models alongside the conventional mathematical models for conducting their trading operation.

With the introduction of electronic trading and the automation that followed has increased the trading volume thus producing a immense amount of data that representing the trades happening in exchanges. Exchanges have been gathering this trading data, creating comprehensive logs of every transaction, selling them to financial institutions that analyze them to discover signals that provide foresight for changes in the market, which can in turn be used by algorithms to make the profitably manage investments. However, applying machine learning techniques on such large-scale data is not a straightforward task. Being able to utilize the information at this scale can provide strategies for many different market conditions but also safeguard from volatile market movements.

The main contribution of this work is the proposal of a deep learning methodology, based on recurrent neural networks, that can be used for predicting future midprice movements from large-scale high-frequency limit order data.

In Section 2 related work on machine learning models that were applied on financial data is briefly presented. Then, the used large-scale dataset is described in detail in Section 3. In Section 4 the proposed deep learning 
methodology is introduced, while in Section 5 the experimental evaluation is provided. Finally, conclusions are drawn and future work is discussed in Section 6.

\section{RELATED WORK}

Recent Deep Learning methods has been shown to significantly improve upon previous machine learning techniques in tasks such as speech recognition [5], image captioning [6], [7], and question answering [8]. Deep Learning models, such as Convolutional Neural Networks (CNNs) [9], and Recurrent Neural Networks (RNNs), e.g., the Long Short-Term Memory Units (LSTMs) [10], have greatly contributed in the increase of performance on these fields, with ever deeper architectures producing even better results [11].

In Deep Portfolio Theory [12], the authors use autoencoders to optimize the performance of a portfolio and beat several profit benchmarks, such as the biotechnology IBB Index. Similarly in [2], a Restricted Boltzmann Machine (RBM) is used to encode monthly closing prices of stocks and then it is fine-tuned to predict the direction the price of each stock will move (above or below the median change). This strategy is compared to a simple momentum strategy and it is established that the proposed method achieves significant improvements in annualized returns.

The daily data of the S\&P 500 market fund prices and Google domestic trends of 25 terms like "bankruptcy" and "insurance" are used as the input to a recurrent neural network that it is trained to predict the volatility of the market fund's price [3]. This method greatly improves upon existing benchmarks, such as autoregressive GARCH and Lasso techniques.

An application using high frequency limit orderbook (LOB) data is [4], where the authors create a set of handcrafted features, such as price differences, bidask spreads, and price and volume derivatives. Then, a Support Vector Machine (SVM) is trained to predict whether the mid-price will move upwards or downward in the near future using these features. However, only 2000 data points are used for training the SVM in each training round, limiting the prediction accuracy of the model.

To the best of our knowledge this is the first work that uses a Limit Order Book data on such a large-scale with more than 4 million events to train LSTMs for predicting the price movement of stocks. The method proposed in this paper is also combined with an intelligent normalization scheme that takes into account the differences in the price scales between different stocks and time periods, which is essential for effectively scaling to such largescale data.

\section{High FReQUency Limit ORder Data}

In financial equity markets a limit order is a type of order to buy or sell a specific number of shares within a set price. For example, a sell limit order (ask) of $\$ 10$ with volume of 100 indicates that the seller wishes to sell the 100 shares for no less that $\$ 10$ each. Respectively, a buy limit order (bid) of $\$ 10$ it means that the buyer wishes to buy a specified amount of shares for no more than $\$ 10$ each.

Consequently the orderbook has two sides, the bid side, containing buy orders with prices $\mathbf{p}_{b}(t)$ and volumes $\mathbf{v}_{b}(t)$, and the ask side, containing sell orders with prices $\mathbf{p}_{a}(t)$ and volumes $\mathbf{v}_{a}(t)$. The orders are sorted on both sides based on the price. On the bid side $p_{b}^{(1)}(t)$ is the is the highest available buy price and on the ask side $p_{a}^{(1)}(t)$ is the lowest available sell price.

Whenever a bid order price exceeds an ask order price $p_{b}^{(i)}(t)>p_{a}^{(j)}(t)$, they "annihilate", executing the orders and exchanging the traded assets between the investors. If there are more than two orders that fulfill the price range requirement the effect chains to them as well. Since the orders do not usually have the same requested volume, the order with the greater size remains in the orderbook with the remaining unfulfilled volume.

Several tasks arise from this data ranging from the prediction of the price trend and the regression of the future value of a metric, e.g., volatility, to the detection of anomalous events that cause price jumps, either upwards or downwards. These tasks can lead to interesting applications, such as protecting the investments when market condition are unreliable, or taking advantage of such conditions to create automated trading techniques for profit.

Methods utilizing this data often use subsampling techniques, such as the OHLC (Open-High-Low-Close) resampling [13], to limit the number of values exist for each timeframe, e.g., every minute or every day. Even though the OHLC method preserves the trend features of the market movements, it removes all the microstructure information of the markets. Note that it is difficult to preserve all the information contained in the LOB data, since orders arrive inconsistently and most methods require a specific number of features for each time step. This is one of the problems RNNs can solve and take full advantage of the information contained in the data, since they can natively handle this inconsistent amount of incoming orders. 


\section{LSTMS FOR FINANCIAL DATA}

The input data consists of 10 orders for each side of the LOB (bid and ask). Each order is described by 2 values, the price and the volume. In total we have 40 values for each timestep. The stock data, provided by Nasdaq Nordic, come from the Finnish companies Kesko Oyj, Outokumpu Oyj, Sampo, Rautaruukki and Wartsila Oyj. The time period used for collecting that data ranges from the 1st to the 14th June 2010 (only business days are included), while the data are provided by the Nasdaq Nordic data feeds [14].

The dataset is made up of 10 days for 5 different stocks and the total number of messages is 4.5 million with equally many separate depths. Since the price and volume range is much greater than the range of the values of the activation function of our neural network, we need to normalize the data before feeding them to the network. To this end, standardization (z-score) is employed to normalize the data:

$$
\mathbf{x}_{\text {norm }}=\frac{\mathbf{x}-\bar{x}}{\sigma_{\bar{x}}}
$$

where $\mathbf{x}$ is the vector of values we want to normalize, $\bar{x}$ is the mean value of the data and $\sigma_{\bar{x}}$ is the standard deviation of the data. Instead of simply normalizing all the values together, we take into account the scale differences between order prices and order volumes and we use a separate normalizer, with different mean and standard deviation, for each of them. Also, since different stocks have different price ranges and drastic distributions shifts might occur in individual stocks for different days, the normalization of the current day's values uses the mean and standard deviation calculated using previous day's data.

We want to predict the direction towards which the price will change. In this work the term price is used to refer to the mid-price of a stock, which is defined as the mean between the best bid price and best ask price at time $t$ :

$$
p_{t}=\frac{p_{a}^{(1)}(t)+p_{b}^{(1)}(t)}{2}
$$

This is a virtual value for the price since no order can happen at that exact price, but predicting its upwards or downwards movement provides a good estimate of the price of the future orders. A set of discrete choices must be constructed from our data to use as targets for our classification model. Simply using $p_{t}>p_{t+k}$ to determine the direction of the mid price would introduce unmanageable amount of noise, since the smallest change would be registered as an upward or downward movement.
Note that each consecutive depth sample is only slightly different from the previous one. Thus the shortterm changes between prices are very small and noisy. In order to filter such noise from the extracted labels we use the following smoothed approach. First, the mean of the previous $k$ mid-prices, denoted by $m_{b}$, and the mean of the next $k$ mid-prices, denoted by $m_{a}$, are defined as:

$$
\begin{aligned}
& m_{b}(t)=\frac{1}{k} \sum_{i=0}^{k} p_{t-i} \\
& m_{a}(t)=\frac{1}{k} \sum_{i=1}^{k} p_{t+i}
\end{aligned}
$$

where $p_{t}$ is the mid price as described in Equation (2). Then, a label $l_{t}$ that express the direction of price movement at time $t$ is extracted by comparing the previously defined quantities $\left(m_{b}\right.$ and $\left.m_{a}\right)$ :

$$
l_{t}=\left\{\begin{array}{cl}
1, & \text { if } m_{b}(t)>m_{a}(t) \cdot(1+\alpha) \\
-1, & \text { if } m_{b}(t)<m_{a}(t) \cdot(1-\alpha) \\
0, & \text { otherwise }
\end{array}\right.
$$

where the threshold $\alpha$ is set as the least amount of change in price that must occur for it to be considered upward or downward. If the price does not exceed this limit, the sample will be considered to belong to the stationary class. Therefore, the resulting label expresses the current trend we wish to predict. Note that this process is applied for every time step in our data.

An improved version of RNNs, namely the LSTM [10], is employed to classify our data. The LSTM solves the problem of vanishing gradients, which makes virtually impossible for an $\mathrm{RNN}$ to learn to correlate temporally distant events. This is achieved by protecting its hidden activation using gates between each of its transaction points with the rest of its layer. The hidden activation that is protected is called the cell state. The following equations describe the behavior of the LSTM model [10]:

$$
\begin{aligned}
\mathbf{f}_{t} & =\sigma\left(\mathbf{W}_{x f} \mathbf{x}+\mathbf{W}_{h f} \mathbf{h}_{t-1}+\mathbf{b}_{f}\right) \\
\mathbf{i}_{t} & =\sigma\left(\mathbf{W}_{x i} \mathbf{x}+\mathbf{W}_{h i} \mathbf{h}_{t-1}+\mathbf{b}_{i}\right) \\
\mathbf{c}_{t}^{\prime} & =\tanh \left(\mathbf{W}_{h c} \mathbf{h}_{t-1}+\mathbf{W}_{x c} \mathbf{x}_{t}+\mathbf{b}_{c}\right) \\
\mathbf{c}_{t} & =\mathbf{f}_{t} \mathbf{c}_{t-1}+\mathbf{i}_{t} \mathbf{c}_{t}^{\prime} \\
\mathbf{o}_{t} & =\sigma\left(\mathbf{W}_{o c} \mathbf{c}_{t}+\mathbf{W}_{o h} \mathbf{h}_{t-1}+\mathbf{b}_{o}\right) \\
\mathbf{h}_{t} & =\mathbf{o}_{t} \sigma\left(\mathbf{c}_{t}\right)
\end{aligned}
$$

where $\mathbf{f}_{t}, \mathbf{i}_{t}$ and $\mathbf{o}_{t}$ are the activations of the input, forget and output gates at time-step $t$, which control how much 
of the input and the previous state will be considered and how much of the cell state will be included in the hidden activation of the network. The protected cell activation at time-step $t$ is denoted by $\mathbf{c}_{t}$, whereas $\mathbf{h}_{t}$ is the activation that will be given to other components of the model.

The parameters of the model are learned by minimizing the categorical cross entropy loss defined as:

$$
\mathcal{L}(\mathbf{W})=-\sum_{i=1}^{L} y_{i} \cdot \log \hat{y}_{i}
$$

where $L$ is the number of different labels and the notation $\mathbf{W}$ is used to refer to the parameters of the LSTM, i.e., $\mathbf{W}_{x f}, \mathbf{W}_{h f}, \mathbf{W}_{x i}, \mathbf{W}_{h i}, \mathbf{W}_{h c}, \mathbf{W}_{x c}, \mathbf{W}_{o c}, \mathbf{W}_{o h}, \mathbf{b}_{f}, \mathbf{b}_{i}$, $\mathbf{b}_{c}$, and $\mathbf{b}_{o}$. The ground truth vector is denoted by $\mathbf{y}$, while $\hat{\mathbf{y}}$ is the predicted label distribution. The loss is summed over all samples in each batch. The most commonly used method to minimize the loss function defined in Equation (12) and learn the parameters W of the model is gradient descent [15]:

$$
\mathbf{W}^{\prime}=\mathbf{W}-\eta \cdot \frac{\partial \mathcal{L}}{\partial \mathbf{W}}
$$

where $\mathbf{W}^{\prime}$ are the parameters of the model after each gradient descent step and $\eta$ is the learning rate. In this work we utilize the Adaptive Moment Estimation algorithm, known as ADAM [16], which ensures that the learning steps are scale invariant with respect to the parameter gradients.

The input to the LSTM is a sequence of vectors $\mathbf{X}=$ $\left\{\mathbf{x}_{0}, \mathbf{x}_{1}, \ldots, \mathbf{x}_{n}\right\}$ that represent the LOB depth at each time step $t$. Each $\mathbf{x}_{t}$ is fed sequentially to the LSTM and its output $\mathbf{y}_{t}$ expresses the categorical distribution for the three direction labels (upward, downward and stationary), as described in Equation (5), for each timestep $t$.

\section{EXPERIMENTAL EVALUATION}

In our first attempt to train an LSTM network to predict the mid-price trend direction we noticed a very interesting pattern in the mean cost per recurrent step, as shown in Figure 1. The cost is significantly higher on the initial steps before it eventually settles. This happens because it is not possible for the network to build a correct internal representation having seen only a few samples of the depth. To avoid this unnecessary source of error and noise in the training gradients, we do not propagate the error for the first 100 recurrent steps. These steps are treated as a "burn-in" sequence, allowing the network to observe a portion of the LOB depth timeline before making an accountable prediction.

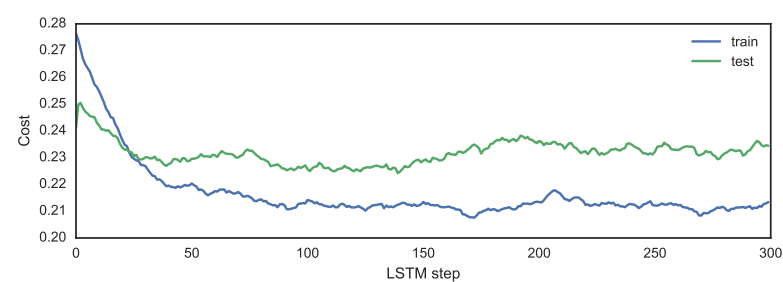

Fig. 1: Mean cost per recurrent step of the LSTM network

TABLE I: Experimental results for different prediction horizons $k$

\begin{tabular}{|c|c|c|c|c|}
\hline Model & Mean Recall & Mean Prec. & Mean F1 & Cohen's $\kappa$ \\
\hline \multicolumn{5}{|c|}{ Prediction Horizon $k=10$} \\
\hline SVM & $39.62 \%$ & $44.92 \%$ & $35.88 \%$ & 0.068 \\
\hline MLP & $47.81 \%$ & $60.78 \%$ & $48.27 \%$ & 0.226 \\
\hline LSTM & $\mathbf{6 0 . 7 7} \%$ & $\mathbf{7 5 . 9 2} \%$ & $\mathbf{6 6 . 3 3} \%$ & $\mathbf{0 . 5 0 0}$ \\
\hline \multicolumn{5}{|c|}{ Prediction Horizon $k=20$} \\
\hline SVM & $45.08 \%$ & $47.77 \%$ & $43.20 \%$ & 0.139 \\
\hline MLP & $51.33 \%$ & $65.20 \%$ & $51.12 \%$ & 0.255 \\
\hline LSTM & $\mathbf{5 9 . 6 0} \%$ & $\mathbf{7 0 . 5 2} \%$ & $\mathbf{6 2 . 3 7} \%$ & $\mathbf{0 . 4 3 0}$ \\
\hline \multicolumn{5}{|c|}{ Prediction Horizon $k=50$} \\
\hline SVM & $46.05 \%$ & $60.30 \%$ & $49.42 \%$ & 0.243 \\
\hline MLP & $55.21 \%$ & $67.14 \%$ & $55.95 \%$ & 0.324 \\
\hline LSTM & $\mathbf{6 0 . 0 3} \%$ & $\mathbf{6 8 . 5 0} \%$ & $\mathbf{6 1 . 4 3} \%$ & $\mathbf{0 . 4 1 1}$ \\
\hline
\end{tabular}

Experimentally we found out that to avoid over-fitting the hidden layer of the LSTM should contain 32 to 64 hidden neurons. If more hidden neurons are used, then the network can easily overfit the data, while if less hidden neurons are used the network under-fits the data reducing the accuracy of the predictions.

We use an LSTM with 40 hidden neurons followed by a feed-forward layer with Leaky Rectifying Linear Units as activation function [17]. We split our dataset as follows. The first 7 days are used to train the network, while the next 3 days are used as test data. We train the same model for 3 different prediction horizons $k$, as defined in Equations (3) and (4).

To measure the performance of our model we use Kohen's kappa [18], which is used to measure the concordance between sets of given answers, taking into consideration the possibility of random agreements happening. We also report the mean recall, precision and F1 score between all 3 classes. Recall is the number true positive samples divided by the sum of true positives and false negatives, while precision is the number of true positive divided by the sum of true positives and false positives. F1 score is the harmonic mean of the precision and recall metrics. 
The results of our experiments are shown in Table I. We compare our results with those of a Linear SVM model and an MLP model with Leaky Rectifiers as activation function. The SVM model is trained using stochastic gradient descent since the dataset is too large to use a closed-form solution. The MLP model uses a single hidden layer with 128 neurons with Leaky ReLU activations. The regularization parameter of the SVM was chosen using cross validation on a split from the training set. Since both models are sequential, we feed the concatenation of the previous 100 depth samples as input and we use as prediction target the price movement associated with the last depth sample. The proposed method significantly outperforms all the other evaluated models, especially for short term prediction horizons $(t=10$ and $t=20)$.

\section{CONCLUSION}

In this work we trained an LSTM network on high frequency LOB data, applying a temporally aware normalization scheme on the volumes and prices of the LOB depth. The proposed approach was evaluated using different prediction horizons and it was demonstrated that it performs significantly better than other techniques, such as Linear SVMs and MLPs, when trying to predict short term price movements.

There are several interesting future research directions. First, more data can be used to train the proposed model, scaling up to a billion training samples, to determine if using more data leads to better classification performance. With more data also increase the "burn-in" phase along with the prediction horizon to gauge the models ability to predict the trend further into the future. Also, an attention mechanism [6], [19], can be introduced to allow the network to capture only the relevant information and avoid noise. Finally, more advanced trainable normalization techniques can be used, as it was established that normalization is essential to ensure that the learned model will generalize well on unseen data.

\section{ACKNOWLEDGMENT}

The research leading to these results has received funding from the H2020 Project BigDataFinance MSCAITN-ETN 675044 (http://bigdatafinance.eu), Training for Big Data in Financial Research and Risk Management. Alexandros Iosifidis was supported from the Academy of Finland Postdoctoral Research Fellowship (No. 295854). He joined Aarhus University on August 2017.

\section{REFERENCES}

[1] M. F. Dixon, D. Klabjan, and J. H. Bang, "Classification-based financial markets prediction using deep neural networks," 2016.
[2] L. Takeuchi and Y.-Y. A. Lee, "Applying deep learning to enhance momentum trading strategies in stocks," 2013.

[3] R. Xiong, E. P. Nichols, and Y. Shen, "Deep learning stock volatility with google domestic trends," arXiv preprint arXiv:1512.04916, 2015.

[4] A. N. Kercheval and Y. Zhang, "Modelling high-frequency limit order book dynamics with support vector machines," Quantitative Finance, vol. 15, no. 8, pp. 1315-1329, 2015.

[5] A. Graves, A.-r. Mohamed, and G. Hinton, "Speech recognition with deep recurrent neural networks," in Proceedings of the IEEE international conference on Acoustics, Speech and Signal Processing (icassp), 2013, pp. 6645-6649.

[6] K. Xu, J. Ba, R. Kiros, K. Cho, A. C. Courville, R. Salakhutdinov, R. S. Zemel, and Y. Bengio, "Show, attend and tell: Neural image caption generation with visual attention." in Proceedings of the International Conference on Machine Learning, vol. 14, 2015, pp. $77-81$.

[7] J. Mao, W. Xu, Y. Yang, J. Wang, Z. Huang, and A. Yuille, "Deep captioning with multimodal recurrent neural networks (m-rnn)," arXiv preprint arXiv:1412.6632, 2014.

[8] Y. Zhu, O. Groth, M. Bernstein, and L. Fei-Fei, "Visual7w: Grounded question answering in images," in Proceedings of the IEEE Conference on Computer Vision and Pattern Recognition, 2016, pp. 4995-5004.

[9] Y. LeCun, Y. Bengio et al., "Convolutional networks for images, speech, and time series," The handbook of brain theory and neural networks, vol. 3361, no. 10, p. 1995, 1995.

[10] S. Hochreiter and J. Schmidhuber, "Long short-term memory," Neural Computation, vol. 9, no. 8, pp. 1735-1780, 1997.

[11] K. He, X. Zhang, S. Ren, and J. Sun, "Deep residual learning for image recognition," in Proceedings of the IEEE Conference on Computer Vision and Pattern Recognition, 2016, pp. 770-778.

[12] J. Heaton, N. Polson, and J. Witte, "Deep portfolio theory," arXiv preprint arXiv:1605.07230, 2016.

[13] D. Yang and Q. Zhang, "Drift-independent volatility estimation based on high, low, open, and close prices," The Journal of Business, vol. 73, no. 3, pp. 477-492, 2000.

[14] M. Siikanen, J. Kanniainen, and J. Valli, "Limit order books and liquidity around scheduled and non-scheduled announcements: Empirical evidence from nasdaq nordic," Finance Research Letters, vol. to appear, 2016.

[15] P. J. Werbos, "Backpropagation through time: what it does and how to do it," Proceedings of the IEEE, vol. 78, no. 10, pp. 1550-1560, 1990.

[16] D. Kingma and J. Ba, "Adam: A method for stochastic optimization," arXiv preprint arXiv:1412.6980, 2014.

[17] A. L. Maas, A. Y. Hannun, and A. Y. Ng, "Rectifier nonlinearities improve neural network acoustic models," in Proceedings of the International Conference on Machine Learning, vol. 30, no. 1, 2013.

[18] J. Cohen, "A coefficient of agreement for nominal scales," Educational and Psychological Measurement, vol. 20, no. 1, pp. 37-46, 1960.

[19] K. Cho, A. Courville, and Y. Bengio, "Describing multimedia content using attention-based encoder-decoder networks," IEEE Transactions on Multimedia, vol. 17, no. 11, pp. 1875-1886, 2015. 